\title{
Viscothermal Effects in a Two-Dimensional Acoustic Black Hole: A Boundary Element Approach
}

\author{
Vicente Cutanda Henríquez $\circledast^{1, *}$ and José Sánchez-Dehesa $\circledast^{2, \dagger}$ \\ ${ }^{1}$ Centre for Acoustic-Mechanical Micro Systems, Technical University of Denmark, Ørsteds Plads, Building 352, \\ Kgs. Lyngby DK-2800, Denmark \\ ${ }^{2}$ Department of Electronic Engineering, Universitat Politècnica de València, Camino de Vera s/n (Building 7F), \\ Valencia ES-46022, Spain
}

(Received 10 February 2021; revised 28 April 2021; accepted 27 May 2021; published 23 June 2021)

\begin{abstract}
The acoustic analog of the quantum black hole for airborne sound in two dimensions was denominated as an omnidirectional acoustic absorber by Climente et al. [see Appl. Phys. Lett., 100, 144103 (2012)], who characterized its absorbing properties without providing any theoretical support. The viscothermal losses of the underlying structure, which consists of an absorbing core and a surrounding gradient-index (GRIN) lens both made of periodic distributions of cylindrical rods, are here comprehensively studied by using the boundary element method (BEM) in two dimensions. It is shown that the numerical simulations in two dimensions reproduce fairly well the increase in absorption of the core when the GRIN lens is added and reveal that the discrepancy between measured and calculated values of absorbance is an artifact of the experimental setup. The possibility of independent calculation of viscous and thermal losses contributions in the two-dimensional (2D) BEM algorithm is employed for the comparison with a homogenization theory in which the cluster of cylinders is represented by a single fluidlike viscous cylinder with effective parameters. We conclude that viscous losses represent about $90 \%$ of the total energy dissipated in the core. The homogenization approach results are only $2 \%$ below the results calculated with $2 \mathrm{D}$ BEM, indicating that the effective parameters obtained by the homogenization are very accurate.
\end{abstract}

DOI: 10.1103/PhysRevApplied.15.064057

\section{INTRODUCTION}

The classical analogs of the quantum hole effect are topics of increasing interest in physics. The photonic black hole $(\mathrm{PBH})$ [1-3] and the acoustic black hole (ABH) [4-11] have been proposed as efficient structures for omnidirectional absorption of electromagnetic and acoustic energies, respectively. The term $\mathrm{ABH}$ was employed by Krylov and Tilman [4] to represent the behavior of flexural waves propagating in a sharp edge that become trapped as they approach the tip. Later on, the concept of an $\mathrm{ABH}$ has been extended to passive vibration control in beams and plates. Though the denomination of "vibration acoustic black hole" would be more appropriate, the term ABH is widely accepted by the scientific community. The vibration control by the ABH effect is out of the scope of this work and readers interested in this particular topic are addressed to the recent review by Pelat and coworkers [12].

Some initial proposals of $\mathrm{ABHs}$ in a fluid background were denominated as omnidirectional acoustic absorbers $[5,6]$ and their performance was supported by numerical

\footnotetext{
*vcuhe@elektro.dtu.dk

†jsdehesa@upv.es
}

simulations. Afterward, Climente et al. [7] reported a practical realization of an $\mathrm{ABH}$ for airborne sound, which was based in lattices of hard cylinders, using a metamaterial core consisting in a highly packed sonic crystal. Later, a similar design was employed by Naify et al. [9] to demonstrate its functionality for underwater sound. On similar backgrounds, Elliot et al. [10] employed a porous material together with a metamaterial matching layer as the absorbing core. Gu and coworkers [11] used angularly distributed fins as the external shell in charge of focusing the acoustic energy towards the absorbing core. Unfortunately, the experimental works did not present any convincing theoretical analysis supporting the absorbing features measured in their respective characterizations.

The study of $\mathrm{ABHs}$ is interesting not only from the fundamental point of view but also for its potential in realistic applications as sinks of environmental noise. The fast development of rapid prototyping machines allows the fabrication of the two-dimensional (2D) $\mathrm{ABH}$ structures previously described, as well as their three-dimensional (3D) counterparts. The ABH can be considered as a proofof-concept of an omnidirectional and broadband absorbing device suitable as a covering structure to arbitrary surfaces where the sound needs be dissipated. 
Here, we are interested in studying the absorbing properties of the ABH characterized in Ref. [7]. The structure consists of an external shell made of cylinders with various radii, which are calculated with the purpose of bending the impinging waves towards the absorbing core. Figure 1 describes the fabricated $\mathrm{ABH}$, the components of which, the external shell and the inner core, are specifically designed acoustic metamaterials based on lattices of rigid cylinders. In Ref. [7], the authors extended previous works on gradient-index (GRIN) acoustic lenses with flat surfaces $[13,14]$ to design a GRIN lens with circular symmetry,
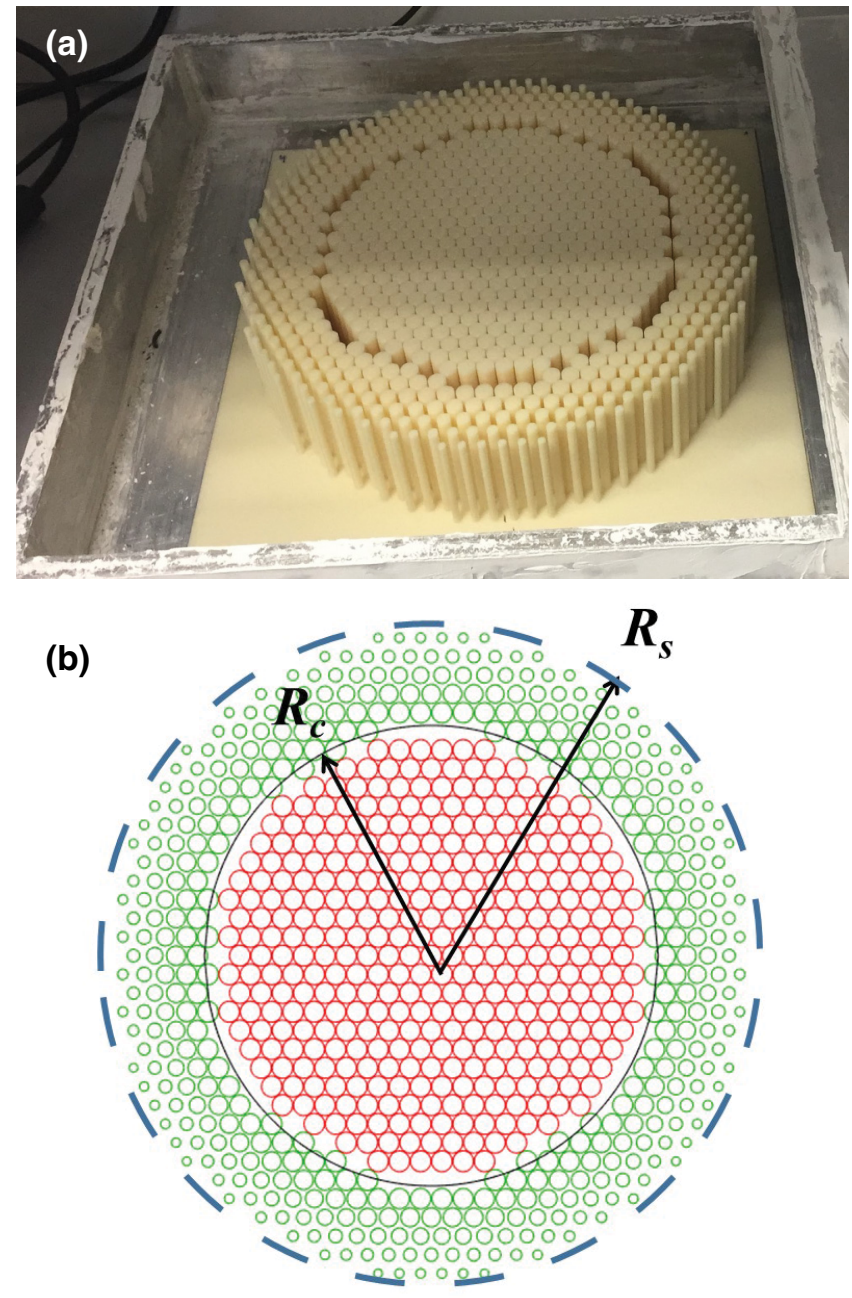

FIG. 1. (a) Photograph of the ABH inside the characterization chamber employed in [7]. (b) Sketch of the ABH, where the outer layer of green circles represents the cylindrical scatterers designed to act as a GRIN lens in charge of redirecting the impinging sound towards the metamaterial core, which is represented by the hexagonal lattice of red circles. The core is designed to absorb the acoustic energy by viscothermal losses arising in the small separation between cylinders. Here $R_{c}$ and $R_{s}$ define the radii of the imaginary circles enclosing the two structures. defining the external shell in charge of energy redirection. The circular GRIN lens plays the role of an acoustic concentrator, a device that has its own interest because it has important applications also for acoustic sensing and energy harvesting [15]. Recently, a 3D acoustic concentrator based on a metamaterial has been demonstrated for airborne sound [16]. On the other hand, a metamaterial core is implemented in [7] using a highly packed sonic crystal, which dissipates the arriving energy due to its viscothermal losses [17]. Though evidence of energy dissipation has been demonstrated experimentally, a calculation of viscothermal effects supporting the recorded data is still lacking.

This work presents a comprehensive study of the viscothermal losses in the structures analyzed in [7]. Particularly, we have employed an improved version of the boundary element method (BEM) [18] implementing the Navier-Stokes equations $[19,20]$ to show how viscothermal effects determine the absorbing features observed in the manufactured ABH [7]. The BEM algorithm was already successfully applied to explain why some types of double-negative metamaterials [21] are not able to exhibit their expected behavior because of losses within the viscous and thermal boundary layers formed around the solid scatterers forming the structure [22].

The study of viscothermal effects on acoustic devices and structures based on lattices of hard scatterers embedded in a fluid, as the $\mathrm{ABH}$, is a topic of increasing interest since the resulting losses ultimately determine their practical functionality [17,23-28]. Remember that losses can, except for long-distance propagation, be ignored in free air or water, but the dissipation is greatly enhanced when sound waves impinge a single hard scatterer due to the existence of thin viscous and thermal boundary layers with thicknesses $\delta_{v}$ and $\delta_{\kappa}$, respectively. Although the dissipation of acoustic energy around a single hard scatterer can be considered low, the amount of losses increases by orders of magnitude, as compared with the losses of the surrounding fluid, when the cylinders are arranged in a lattice and the fraction of scatterers $f$ increases [29]. The absorbing characteristics of continuously graded phononic crystals has been pointed out previously [30]. More recently, the relevance of viscous dissipation has been analyzed numerically for the case of a tubular phononic crystal sensor [31].

The extraordinary properties of acoustic metamaterials appear in the long-wavelength limit where the lattices of scatterers behave like a uniform medium with effective parameters (mass density and bulk modulus), sometimes negative, which can be obtained using different homogenization theories [32-36].

A comparison with the measurements published in [7] indicates that our BEM simulations give a good qualitative description of the absorbing features of the $\mathrm{ABH}$ and the fundamental role played by the GRIN lens shell 
in order to enhance the dissipating properties of the core. Discrepancies between measured and calculated values in the absorbance spectra have been attributed to the intrinsic losses of the characterization chamber employed in the experimental setup.

In addition, the viscous and thermal dissipation in the metamaterial structure defining the core have been independently analyzed in order to assess the contribution of viscous losses to its total absorption. Thus, dissipating losses due to viscosity are calculated for the cluster of cylinders defining the bare core (BC). The results are compared with that obtained by representing the cluster as a uniform viscous cylinder with effective parameters, including a frequency-dependent imaginary part for the wave number. The BEM simulations indicate that the effective viscous cylinder reproduces fairly well the absorbance spectra obtained when considering the full cluster geometry and show that viscous losses explain about $90 \%$ of the total energy dissipated by viscothermal effects in this setup.

This article is organized as follows. After this introduction, Sec. II describes the features of the ABH under study and explains the experimental approach employed in its characterization. A brief description of the 2D BEM employed in the numerical simulations of the $\mathrm{ABH}$ and related structures is also presented. Afterward, Sec. III reports the results from the BEM simulations, which are discussed and compared with measurements reported in [7]. The absorbance spectra for the $\mathrm{BC}$ and the isolated GRIN lens are studied separately in Sec. III A, which presents not only the numerical simulations but also the corresponding experimental spectra. On the other hand, Sec. III A presents the study of viscous losses in clusters with inner structure similar to the BC. The computational demand to solve these structures with the 2D BEM has been reduced dramatically thanks to the introduction of an homogenization approach, which allows the cluster to be represented with a single uniform cylinder. It is shown that the homogenized cylinder describes fairly well the absorbing features of the exact clusters and facilitates the study of the viscous absorbance as a function of the cluster dimensions. A summary and conclusions are presented in Sec. IV.

\section{THEORETICAL APPROACH: THE BOUNDARY ELEMENT METHOD}

The structure of the $\mathrm{ABH}$ under study is shown in Fig. 1, where Fig. 1(a) shows a photo inside the characterization chamber and Fig. 1(b) depicts a schematic view, where the circular sections of cylinders are colored to distinguish between the two components of the ABH. The green circles define the GRIN lens component, which is made of 408 cylinders distributed in five layers, with radii gradually decreasing with the distance to the center in order to approximately match the acoustic impedance of both the inner core and the surrounding air. The red circles define the inner core, the absorbing component of the $\mathrm{ABH}$, which contains 361 cylinders distributed in a hexagonal lattice with period $a=7.5 \mathrm{~mm}$. All the cylinders have equal diameter, $d_{c}=7.2 \mathrm{~mm}$, corresponding to a lattice filling fraction of $83.6 \%$ [7]. Note that the closepacking $(\mathrm{CP})$ condition for the hexagonal lattice is $90.6 \%$. Therefore, the inner core is a sonic crystal with external shape approximately circular. It can be also considered as a metamaterial designed to dissipate the acoustic energy by viscothermal losses produced in the narrow channels existing between nearest-neighbor cylinders. The actual values of $R_{c}$ and $R_{s}$ in Fig. 1 are 80 and $120 \mathrm{~cm}$, respectively.

Experimentally, the absorbing properties of the $\mathrm{ABH}$ are characterized in a homemade 3D multimode impedance chamber (MMIC), which is schematically depicted in Fig. 2(a). The experimental setup also involves a speaker (red circle) centered at the left-hand-side plate of the MMIC and two arrays of nine microphones (blue dots) placed at the middle of the chamber. The setup requires an additional microphone which is used as a reference [Ref. Mic. in Fig. 1(a)]. The MMIC is manufactured with aluminum plates $1 \mathrm{~cm}$ thick. The bottom and top surfaces are rectangular with the same dimensions. However, the
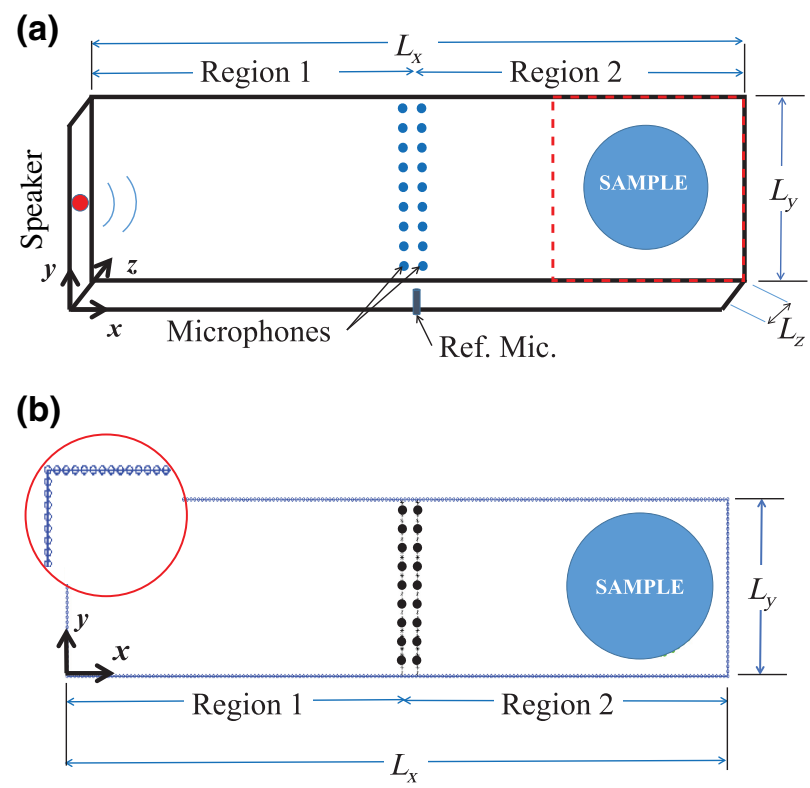

FIG. 2. (a) Sketch of the 3D multimodal impedance chamber employed in the experimental setup. The actual dimensions are [7]: $L_{x}=148 \mathrm{~cm}, L_{y}=29.6 \mathrm{~cm}$, and $L_{z}=5 \mathrm{~cm}$. (b) Sketch of the $2 \mathrm{D}$ chamber employed in the BEM simulations. The chamber boundaries are determined by 212 quadratic three-node line elements. The blue circles (more clearly seen in enlarged view) indicate the positions of the 424 nodes defining the elements. The symbols at the middle of the chambers represent the two rows of points where the pressure waves are captured. 
top surface is made of two plates with different areas. The smaller plate, represented by the red dashed square in Fig. 2(a), is removable to allow the introduction of samples inside the chamber. The dimensions reported in the caption of Fig. 2 correspond to the space available inside the chamber for sound propagation. For a complete description of the characterization procedure the reader is addressed to [7].

For the theoretical model, the 3D problem has been reduced to a $2 \mathrm{D}$ problem because we are always working with frequencies below the frequency cutoff defining the excitation of modes with a nodal line along the $z$ direction (see Appendix A). The numerical simulations have been performed using the BEM instead of the finite element method (FEM) because of its computational advantages for large acoustic setups with losses [19].

Figure 2(b) depicts the section of the cylindrical cavity employed in the 2D BEM simulations. It has been defined with the dimensions reported in Fig. 2(a) for the actual MMIC. Therefore, sound waves, which are generated in the left-hand side, travel along the positive $x$ axis, impinge the sample, and are reflected back by the rigid boundary at the right-hand side. In addition, the pressure field $P(x, y)$ presents acoustic modes involving the $y$ axis finite dimension. In other words,

$$
P(x, y)=\sum_{m=0}^{M}\left[A_{m} e^{i \beta_{m} x}+B_{m} e^{-i \beta_{m} x}\right] \cos \left(\frac{m \pi}{L_{y}} y\right),
$$

where the propagation constants are $\beta_{m}=$ $\sqrt{\omega^{2} / c_{b}^{2}-\left(m \pi / L_{y}\right)^{2}}$, with $\omega=2 \pi v$ the angular frequency, $c_{b}$ the speed of sound in air, and $L_{y}$ the chamber width along $y$. Note that the summation involves only three terms, corresponding to even modes $m=0,2$, and 4 in the $y$ direction. These modes are the only ones excited in the range of frequencies analyzed experimentally [7]. See Appendix A for a discussion of the normal modes excited in the MMIC.

Coefficients $A_{m}$ and $B_{m}$ are obtained experimentally by measuring the pressure with nine pairs of microphones evenly distributed along the $y$ direction, with $3 \mathrm{~cm}$ being the distance between the two rows of microphones along $x$ [see Fig. 2(a)]. However, in the present numerical simulations, the pressure is sampled at 29 position pairs, $\left(x_{\alpha}, y_{\alpha}\right)$, with a separation between rows equal to that employed in the experiments. As the launched waves are deterministic (no reference microphone is needed), the coefficients $A_{m}$ and $B_{m}$ are obtained by solving the following overdetermined system of equations,

$$
P_{\alpha}\left(x_{\alpha}, y_{\alpha}\right)=\sum_{m=0}^{M}\left[A_{m} e^{i \beta_{m} x_{\alpha}}+B_{m} e^{-i \beta_{m} x_{\alpha}}\right] \cos \left(\frac{m \pi}{L_{y}} y_{\alpha}\right),
$$

where $\alpha$ runs from 1 to 58 .

Once the coefficients $A_{m}$ and $B_{m}$ are calculated, the flux $\Phi_{A}$ that enters in region 1 and the flux $\Phi_{B}$ reflected backwards to region 2 are calculated by integration

$$
\begin{aligned}
& \Phi_{A}=\frac{L_{y}}{4 \omega \rho_{b}} \sum_{m=0}^{M} \beta_{m}\left|A_{m}\right|^{2}, \\
& \Phi_{B}=\frac{L_{y}}{4 \omega \rho_{b}} \sum_{m=0}^{M} \beta_{m}\left|B_{m}\right|^{2} .
\end{aligned}
$$

The reflectance is obtained as $\mathcal{R}=\Phi_{B} / \Phi_{A}$ and the absorbance or energy absorbed by the structure placed at region 2 is

$$
\alpha(\omega)=1-\mathcal{R} .
$$

The absorbance is the key parameter characterizing the frequency dependence of the energy dissipated by a given structure.

The comparison of different absorbing structures is performed by using the so-called absorption quality factor $Q_{\alpha}$, which condenses in a single value the absorbing power in a selected band of frequencies $\Delta v$,

$$
Q_{\alpha} \equiv \frac{1}{\Delta v} \int_{v_{i}}^{v_{f}} \alpha(v) d v
$$

where $\Delta v=v_{f}-v_{i}$ is the bandwidth in Hertz. For a fair comparison with the experimental data, values of $Q_{\alpha}$ are calculated using the same range of frequencies, that is, from $v_{i}=580 \mathrm{~Hz}$ to $v_{f}=3400 \mathrm{~Hz}$.

\section{A. Viscothermal effects in the boundary element method}

The BEM with viscothermal losses has been already employed by these authors in previous works $[19,20]$. In brief, the BEM with losses solves the linearized NavierStokes equations with no flow. The three components of the sound field (i.e., the acoustic, thermal, and viscous) are separated and only coupled at the domain boundaries $[37,38]$. Assuming the time dependence $e^{-i \omega t}$, where $\omega$ is the angular frequency, the harmonic equations are

$$
\begin{aligned}
& \left(\Delta+k_{a}^{2}\right) p_{a}=0, \\
& \left(\Delta+k_{h}^{2}\right) p_{h}=0, \\
& \left(\Delta+k_{v}^{2}\right) \vec{v}_{v}=\overrightarrow{0}, \quad \text { with } \Delta \cdot \vec{v}_{v}=0,
\end{aligned}
$$

where indexes $a, h$, and $v$ define the acoustic, thermal, and viscous components, respectively. The total pressure, $p$, is the sum of its acoustic and thermal components because the viscous velocity has no pressure associated: 
$p=p_{a}+p_{h}$. The particle velocity field is the superposition of the three components, $\vec{v}=\vec{v}_{a}+\vec{v}_{h}+\vec{v}_{v}$,

Viscous and thermal components take nonnegligible values only within the so-called viscous and thermal boundary layers with thicknesses, $\delta_{v}$ and $\delta_{\kappa}$, which are inversely proportional to the square root of frequency [39]:

$$
\begin{aligned}
\delta_{v} & =\sqrt{2 \eta / \rho_{0} \omega}, \\
\delta_{\kappa} & =\sqrt{2 \kappa / C_{p} \rho_{0} \omega},
\end{aligned}
$$

where $\eta$ is the viscosity coefficient, $\rho_{0}$ is the fluid density, $\kappa$ is the thermal conductivity, and $C_{p}$ is the specific heat at constant pressure. At audible frequencies, both layers $\delta_{v}$ and $\delta_{\kappa}$ have similar thickness, with values that span from a fraction of a millimeter to a few micrometers [37]. The viscous and thermal losses are usually taken into account as an acoustic impedance of the boundary, and the sound field can be described by the lossless wave equation [40].

In 3D setups, the five unknowns in Eqs. (7a), (7b), and (7c) (i.e., $p_{a}, p_{h}$, and the three components of $\vec{v}_{v}$ ) are calculated by imposing the boundary condition on the total pressure, $p$, and the total velocity $\vec{v}$ [20]. In the 2D implementation employed in this paper, only two components of $\vec{v}_{v}$ are considered, giving four unknowns.

As Eqs. (7a)-(7c) are formally equivalent to the Helmholtz wave equation, the practical implementation of BEM is based on the research software OpenBEM, which solves the Helmholtz wave equation using the direct collocation technique [41]. The BEM algorithm only meshes the domain boundary, saving degrees of freedom as compared with FEM. However, the BEM coefficient matrices are frequency dependent and fully populated, which may counterbalance the mesh reduction. In the case of BEM with viscous and thermal losses, three sets of coefficient matrices are used, corresponding to the three components: acoustic, thermal, and viscous. The thermal and viscous coefficient matrices are usually sparse due to the short reach of such effects, as compared with the overall dimensions of the setup.

Figure 2(b) depicts the mesh defining the cavity boundaries, containing 212 linear three-node triangular surface elements, and the blue dots represent the corresponding 424 nodes. Figures 3(a) and 3(b) show, a quarter of the cylinders defining the $\mathrm{ABH}$ and the structure here denominated as expanded core (EC). Note that the EC is constructed with the same number of cylinders than the $\mathrm{ABH}$ but all the cylinders have equal size. Simulations have been performed by changing the number of quadratic three-node line elements per cylinder: three, six, and twelve elements. As the numerical results for six elements per cylinder are close to those obtained with denser meshes, in what follows, all the results here reported correspond to BEM simulations using six quadratic three-node line elements per cylinder. Therefore, the number of nodes needed to

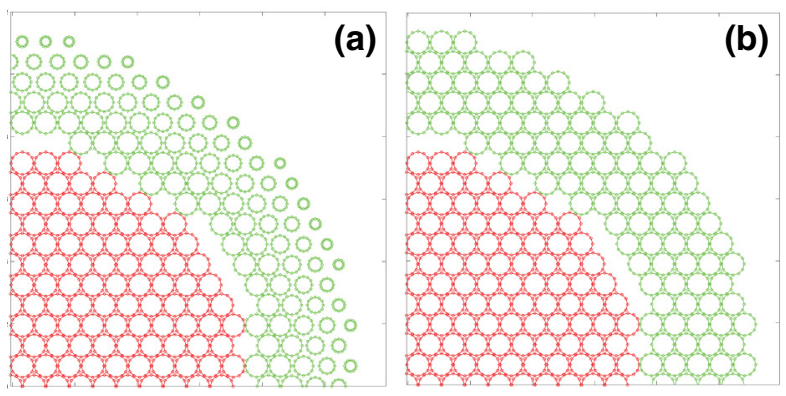

FIG. 3. Sketch representing one quadrant of two structures characterized experimentally with the mesh employed in the BEM simulations: (a) the $\mathrm{ABH}$, made of a GRIN lens (green color) and an absorbing core (red color); (b) the EC, where the cylinders in the GRIN lens are replaced by equal radii cylinders. Quadratic three-node line elements are used to define the cylinders' boundaries. The nodes are represented by the small circles along the boundaries of each cylinder.

describe both the $\mathrm{ABH}$ and the $\mathrm{EC}$ is 9228 , from which 4332 correspond to the core and 4896 correspond to the external shell. A total of 289 frequencies per structure are calculated in the range of frequencies from $400 \mathrm{~Hz}$ to 3400 $\mathrm{Hz}$, with a spacing of $10.4 \mathrm{~Hz}$.

\section{RESULTS AND DISCUSSION}

Figure 4 shows the calculated coefficients $A_{m}$ and $B_{m}$ employed in the linear expansion described by Eq. (2). They correspond to the case of the ABH sample located inside the 2D chamber depicted in Fig. 2(b). In principle, they should be quasi-continuous functions of the

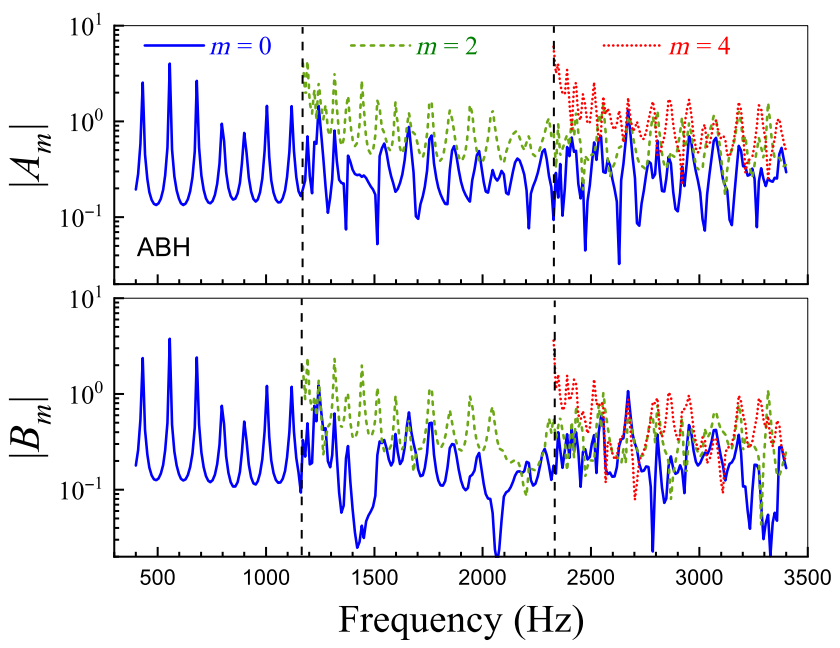

FIG. 4. Modulus of coefficients $A_{m}$ and $B_{m}$ employed in the calculation of the absorbance of the $\mathrm{ABH}$ (see Fig. 1). They are calculated using the BEM algorithm with viscothermal losses. The vertical dashed lines located at 1162 and $2324 \mathrm{~Hz}$ define the cutoff frequencies of modes with $m=2$ (green dashed lines) and $m=4$ (red dotted lines), respectively. 
frequency, which would happen if the length $L_{x}$ of the rectangular cavity is very large. However, the actual length of the cavity along the $x$ axis is not long enough and, therefore, the frequency-dependent coefficients $A_{m}$ and $B_{m}$ show well-defined peaks, as it is also observed in the measured coefficients (see Fig. 4 in [7]). The peaks define the frequencies of the standing waves in the finite 2D box defined by the chamber and the $\mathrm{ABH}$ sample located inside. The reader is addressed to Appendix A, where $A_{m}$ and $B_{m}$ are calculated for the case of the empty chamber and where it is discussed how the frequencies of the peaks change with the size of the sample located at its interior.

Let us remark that the frequency dependence of the ABH coefficients obtained from measurements is well reproduced by the profiles of the calculated coefficients using a 2D approach, which are depicted in Fig. 4. For example, coefficients associated to modes with $m=0$ (blue lines) show equidistant peaks below the onset of modes $m=0$, as the experimental data does. Note also that some particular features in the measured coefficients are well reproduced in the 2D BEM simulations. Thus, $\left|B_{0}\right|$ shows strong minimums at positions 1420, 2067, and 3320 $\mathrm{Hz}$, which are also shown in the reported data but located a slightly shifted frequencies. Overall, it can be concluded that the profiles of the calculated and measured coefficients show a good qualitative agreement. Regarding quantitative discrepancies, they possibly appear due to different issues in the experimental setup as is discussed in the following.

Figures 5(a) and 5(b) represent the absorbance spectra of the $\mathrm{ABH}$ and the $\mathrm{EC}$, respectively. From the comparison

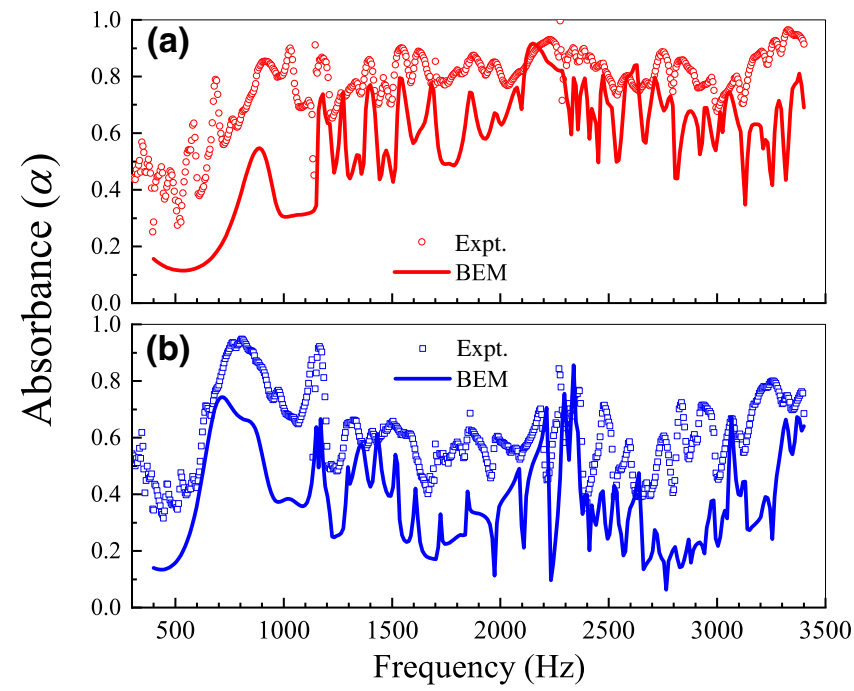

FIG. 5. (a) Calculated absorbance spectrum of the ABH using the BEM algorithm with viscothermal losses (red continuous line). (b) Calculated spectrum (blue continuous line) corresponding to the denominated EC depicted in Fig. 3(b). Their corresponding experimental spectra (hollow symbols) are taken from [7].

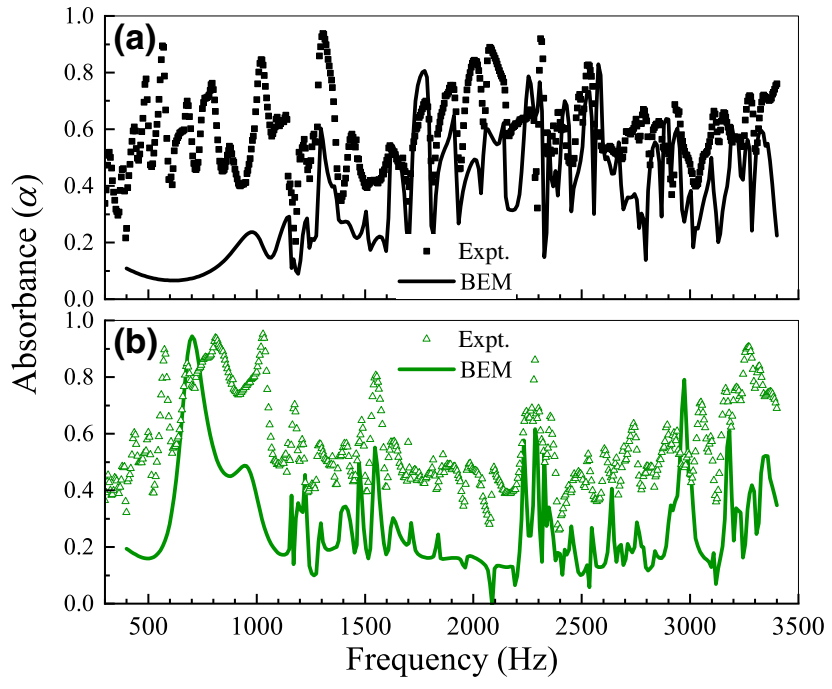

FIG. 6. (a) Absorbance spectrum of the BC calculated with the BEM algorithm (black line). (b) The absorbance spectrum for the shell (green line). The corresponding experimental data (symbols) are taken from Ref. [7].

of both the measured (symbols) and the simulated (lines) spectra, it is concluded that they show a general qualitative agreement in their profiles' shapes, however, strong disagreements are also observed regarding their absolute values. The absorbance values in the experimental spectra are always higher than those obtained using the BEM algorithm. For frequencies below $1200 \mathrm{~Hz}$, the disagreement between experiments and simulations is particularly strong, showing discrepancies not only in the absolute values but also in the profile shape. For higher frequencies, both spectra seem to follow the same profile shape but the simulations predict values smaller than that measured in the actual MMIC.

The absorbing properties of the two components forming the $\mathrm{ABH}$ taken as individual units (i.e., the $\mathrm{BC}$ and the circular GRIN lens) are also studied numerically and compared with the experiments. Figures 6(a) and 6(b) show the resulting spectra for the BC and the GRIN lens shell, respectively. BEM simulations (continuous lines) are compared with measurements (symbols). The discrepancies observed between theoretical and experimental spectra data are similar to those described regarding Fig. 5.

The disagreements between simulations and measurements shown in Figs. 5 and 6 can be understood by considering that the homemade 3D chamber employed in the experimental setup has intrinsic losses adding up to the absorbing properties of the structures characterized inside. This effect is supported by the results shown in Appendix B, which reports the absorbance spectra measured for the empty 3D chamber. Therefore, the discrepancies between theoretical and experimental spectra are attributed to an artifact of the characterization setup, which 


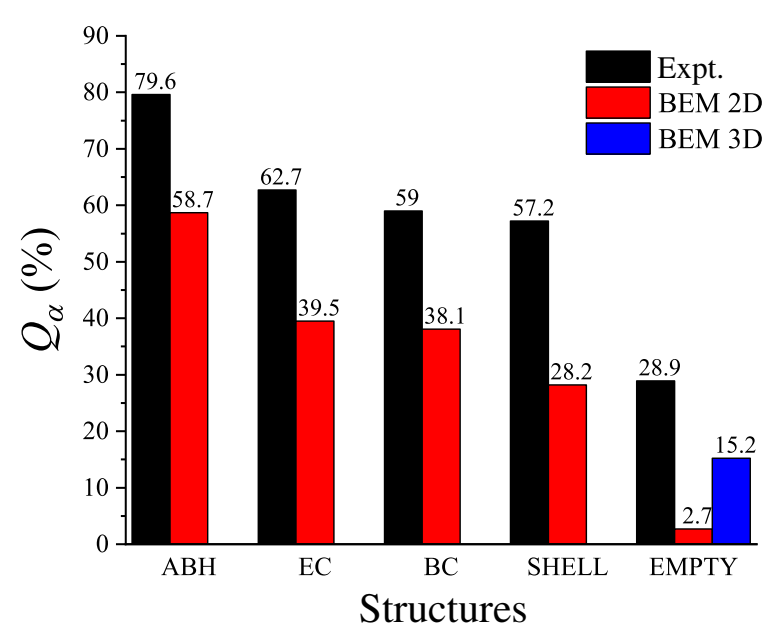

FIG. 7. Absorptive quality factor $Q_{\alpha}$ of the structures under study. Experimental results (black columns) are compared with BEM 2D simulations (red columns) for the $\mathrm{ABH}$, the $\mathrm{EC}$, the $\mathrm{BC}$, and the isolated GRIN shell (SHELL). The case where the impedance chamber has no object (EMPTY) also shows the result obtained with BEM 3D simulations (blue column).

added extra losses to the absorbing features of the samples due to the imperfect manufacturing of the $3 \mathrm{D}$ chamber. A further analysis of the absorbing properties has been performed by calculating the absorption quality factor, $Q_{\alpha}$, described by Eq. (6). Figure 7 shows a comparison between the values $Q_{\alpha}$ obtained from measurement and BEM 2D simulations for the different structures. They are represented in a bar plot. The values corresponding to the $\mathrm{ABH}$ and the $\mathrm{EC}$ are calculated using the spectra shown in Fig. 5. In a similar manner, the values for the bare core (BC) and the isolated GRIN lens shell are obtained with the spectra represented in Fig. 6. The values corresponding to the EMPTY case (i.e., when the chamber has no structure inside) are obtained using the measured spectrum shown in Appendix B and, regarding simulations, the values are obtained by applying the BEM to the $2 \mathrm{D}$ and $3 \mathrm{D}$ chambers shown in Fig. 2.

We observe how the calculated viscothermal factor using the 2D approach, of about $2.7 \%$, is small in comparison with that obtained using full 3D simulations (15.2\%) and very small in comparison with that obtained experimentally, of about $28.9 \%$. As the viscothermal losses in the empty chambers are, in principle, only due to dissipation on its walls, the large value obtained from measurements confirms our previous discussion regarding the undesired extra losses involved in the manufactured empty chamber. This finding is key to understand the discrepancies between calculated and experimental quality factors. Therefore, the value $Q_{\alpha}$ of a given structure that results from the 2D BEM simulations includes, in fact, a small percentage $(2.7 \%)$ associated with the viscothermal absorption taking place at the four walls enclosing the chamber. However, from the experimental point of view, the contribution of the actual 3D chamber is extraordinarily high and cannot be attributed solely to the dissipation on the walls, which according to our 3D BEM simulation is only $15.2 \%$.

In spite of the poor agreement observed in Fig. 7 between calculated and experimental $Q_{\alpha}$ values, interesting conclusions can be drawn from their analysis. For example, the simulations predict that the $Q_{\alpha}$ of the $\mathrm{ABH}$ is $58.7 \%$, indicating that the absorbing power of the $\mathrm{BC}$ increases $19.1 \%$ when it is surrounded with the GRIN lens shell. Experimentally, the increase in $Q_{\alpha}$ is $20.6 \%$. Therefore, the 2D BEM simulations with viscothermal losses underestimated by only $1.5 \%$ the increase in $Q_{\alpha}$ observed experimentally. Taking into account the uncertainties discussed above regarding the experimental setup, we can conclude that the present $2 \mathrm{D}$ simulations are helpful in order to understand the measured data and support the claim that the $\mathrm{ABH}$ responds to the $2 \mathrm{D}$ model employed in its design.

\section{A. Viscous absorption of circular clusters: homogenization approach}

This section is devoted to showing that viscous dissipation arising in clusters with high filling fractions can be reproduced fairly well by using a uniform fluidlike viscous cylinder with effective parameters determined with a two-step procedure. In the first step, the dimension of the cylinder, its mass density, and its bulk modulus are determined by applying the homogenization theory introduced for inviscid cylinders [34,35]. In the second step, the viscous dissipation is represented with an effective imaginary part added to the wave number [29]. This procedure allows the viscous quality factor of clusters to be obtained as a function of the number of cylinders. This enables a simplified numerical calculation, here implemented in BEM, where a given cluster of hard cylinders is replaced by a single fluidlike cylinder with effective viscous parameters.

The homogenization theory [34,35] establishes that effective parameters are valid for large enough clusters made of isotropic lattices of cylinders, like the hexagonal and square lattices. Moreover, it has been also demonstrated than the homogenization approach is valid for wavelengths $\lambda \geq 4 a$, with $a$ being the lattice period of the cylinders' distribution. For the case of clusters made of a small number of cylinders, it has been shown that the homogenization is still applicable to certain magic clusters [42] in which the number of cylinders and their external shape follow some constraints.

As an example, let us consider the structure named as cluster A, consisting of a circular cluster made of 361 cylinders, all with the same diameter $d_{c}=7.2 \mathrm{~mm}$ and distributed in a hexagonal lattice with period $a=7.5 \mathrm{~mm}$. This cluster corresponds to the BC studied in the previous 
section and its viscothermal dissipation, in terms of $Q_{\alpha}$, is already calculated by using the time-consuming $2 \mathrm{D}$ BEM algorithm described in Sec. II. In what follows, we describe how to obtain the effective parameters of the uniform fluidlike cylinder representing the cluster $\mathrm{A}$ and how the effective cylinder accurately reproduces the viscous absorptive properties of the cluster.

The theory predicts [34] that cluster A can be replaced by an homogeneous fluidlike cylinder with the same scattering properties only if the impinging sound waves have wavelengths larger than $4 a=31.2 \mathrm{~mm}$, which corresponds to frequencies below $11 \mathrm{kHz}$. This is satisfactorily accomplished for cluster A because we are interested in its properties up to $3.5 \mathrm{kHz}$. The effective parameters (i.e., radius, mass density, and bulk modulus) of the homogeneous cylinder representing cluster A are determined as follows.

To calculate the effective radius, we use the general expression introduced for the case of a cluster made of $N$ rigid cylinders distributed in a hexagonal lattice [34]:

$$
R_{\mathrm{eff}}=\left(\frac{N \sqrt{3}}{2 \pi}\right)^{1 / 2} a,
$$

which is obtained by the condition of conservation of filling fraction, that is, $f=f_{\text {hex }}$, where $f$ is the volume occupied by the cylinders in the cluster and $f_{\text {hex }}$ is the corresponding value calculated for cylinders distributed in an infinite hexagonal lattice. As $f_{\text {hex }}=(2 \pi / \sqrt{3})\left(d_{c} / 2 a\right)^{2}$, for the parameter of cluster $\mathrm{A}, f_{\text {hex }}=0.836$ and, consequently, $R_{\text {eff }}=74.8 \mathrm{~mm}$, a value slightly smaller than the radius of the imaginary circle enclosing the cluster. The corresponding effective mass density and effective sound speed, obtained from the semianalytical expressions in [35], are

$$
\begin{aligned}
& \rho_{\text {eff }}=17.19 \mathrm{~kg} / \mathrm{m}^{3}, \\
& c_{\text {eff }}=223.48 \mathrm{~m} / \mathrm{s} .
\end{aligned}
$$

As the second step in the homogenization procedure, the energy dissipation occurring inside the cluster due to viscous losses is represented by an effective imaginary part added to the wave number [29]. In other words, it is considered that the pressure propagating in a infinite lattice of cylinders follows the simple expression,

$$
p(x) \approx e^{-\beta_{\mathrm{eff} x}},
$$

where $\beta_{\text {eff }}$ is the decay coefficient for a wave propagating in a fluidlike dissipating medium.

In a viscous fluid, such as air or water, the decay coefficient $\beta_{0}$ grows quadratically with $\omega$ as $\beta_{0}=$ $\left(\omega^{2} / 2 \rho c^{3}\right)\left[\frac{4}{3} \eta+\xi\right]$, where $c$ is the speed of sound, $\rho$ is the fluid density, and $\eta, \xi$ are two viscosity coefficients. For the case of a lattice of hard cylindrical scatterers,

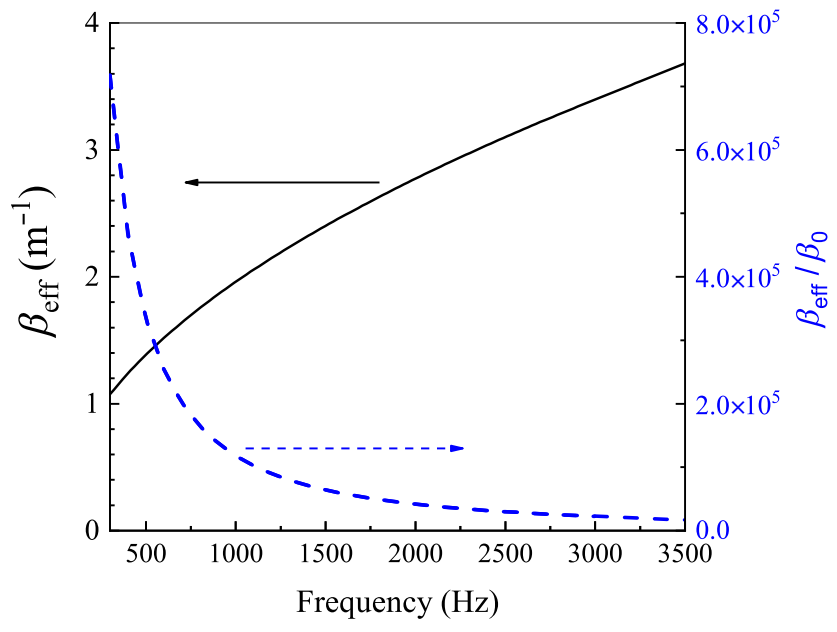

FIG. 8. Calculated decay coefficient, $\beta_{\text {eff }}$, for sound waves propagating in a hexagonal lattice of hard cylinders embedded in air (continuous line). The lattice period is $7.5 \mathrm{~mm}$ and the diameter of the cylinders is $7.2 \mathrm{~mm}$. The blue dashed line represents the coefficient normalized to that of air, $\beta_{0}$, which has been calculated with the following parameters: $\rho_{\text {air }}=1.29 \mathrm{~kg} / \mathrm{m}^{3}$, $c_{\text {air }}=343.98 \mathrm{~m} / \mathrm{s}, \quad \xi=1.92 \times 10^{-5} \mathrm{Ns} / \mathrm{m}^{2}$, and $\eta=18.5 \times$ $10^{-6} \mathrm{Ns} / \mathrm{m}^{2}$.

however, the decay coefficient $\beta_{\text {eff }}$ lies within the interval $\beta_{0} \ll \beta_{\text {eff }} \ll 1 / a$ and, therefore, the calculation of $\beta_{\text {eff }}$ is made possible in the lowest approximation over $\xi$ and $\eta$ (Ref. [29]).

Figure 8 depicts the isotropic decay coefficient $\beta_{\text {eff }}$ (continuous line) resulting from the numerical solution of Eq. (13) in [29]. It is solved for the case of the hexagonal lattice embedded in cluster A. The normalized values (dashed line) indicate that, in the range of frequencies of interest, the sound waves decay much faster in the lattice than in air. In fact, the decay length increases in about five orders of magnitude in comparison with that in air.

In this second step of the homogenization procedure, the effective parameters already calculated (i.e., sound speed and mass density) keep their values since they are viscosity-independent [36].

For the sake of comparison, a larger structure denominated as cluster B has been considered. It is made of 817 cylinders and its calculated effective radius is 112.6 $\mathrm{mm}$, the other effective parameters being equal to those of cluster A. Cluster B is particularly interesting because the cylinders in this cluster occupy a region approximately circular with the same dimension than the ABH. Figures 9(a) and 9(b) show the viscous absorbance spectra calculated for clusters A and B, respectively. The spectra associated to the homogenized clusters (continuous lines) are calculated using the 2D BEM algorithm applied to a single fluidlike cylinder with acoustic parameters determined as explained before. For comparison purposes, Fig. 9 also shows the spectra calculated for the full clusters (dashed lines) by 


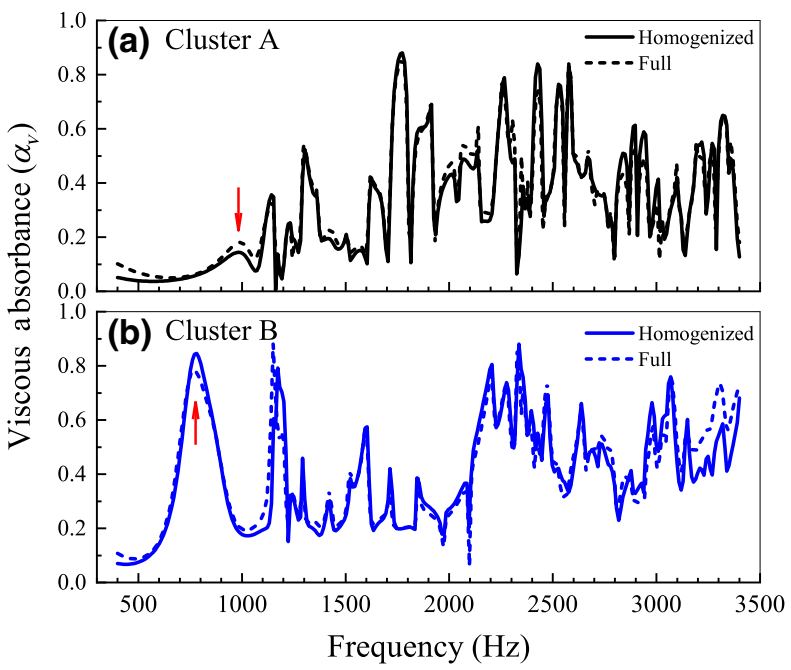

FIG. 9. Calculated viscous absorbance, $\alpha_{v}$, for the structures with parameters described in Table I: (a) cluster A (bare core); (b) cluster B. Results obtained by applying the BEM algorithm to single fluidlike homogenized cylinders (continuous lines) are compared with those obtained using the exact clusters (dashed lines). The arrows indicate the frequencies of the corresponding fundamental modes.

running the BEM algorithm without thermal losses. The absorbance profiles calculated for the homogenized clusters exhibit a perfect agreement with the profiles obtained for the complete clusters, giving strong support to the frequency-dependent effective viscosity developed in the homogenization theory.

Table I reports the absorptive factors due to viscosity, $Q_{\alpha}^{v}$, and their comparison with the corresponding factors due to viscothermal losses, $Q_{\alpha}$. Note how the good agreement between spectral profiles translates into a corresponding good agreement between the quality factors obtained from them. The cluster $\mathrm{C}$ appearing in the last row corresponds to the structure denominated EC, schematically shown in Fig. 3(b). This cluster has the same external

TABLE I. Absorptive factors due to viscous losses only, $Q_{\alpha}^{v}$. BEM values calculated using the full clusters are compared with those calculated using a single fluidlike viscous cylinder with effective parameters. All the clusters have the cylinders enclosed in a circular region. Cluster $\mathrm{C}$ corresponds to the $\mathrm{EC}$ described in Fig. 3(b). The last column gives the contribution of viscous losses in relation to the total viscothermal absorptive factor, $Q_{\alpha}$.

\begin{tabular}{lcccc}
\hline \hline Structure & $\begin{array}{c}\text { Cylinders } \\
\text { (number) }\end{array}$ & $\begin{array}{c}R_{\text {eff }} \\
(\mathrm{mm})\end{array}$ & $Q_{\alpha}^{v}$ & $\begin{array}{c}Q_{\alpha}^{v} / Q_{\alpha} \\
(\%)\end{array}$ \\
\hline Cluster A (full) & 361 & 80 & 33.9 & 90 \\
Cluster A (homogenized) & 1 & 74.8 & 33.4 & 88 \\
Cluster B (full) & 817 & 120 & 42.4 & 91 \\
Cluster B (homogenized) & 1 & 112.6 & 41.5 & 89 \\
Cluster C & 769 & 120 & 35.8 & 91 \\
\hline \hline
\end{tabular}

shape than cluster B but contains a lower number of cylinders. Therefore, the homogenization approach is not applicable due to the existence of vacancies inside the structure. Note that the calculated viscous factor has decreased by about $6 \%$ due the smaller number of cylinders in cluster $\mathrm{C}$ in comparison with cluster B. Finally, the last column in Table I reports the relative contribution of viscous losses to the total viscothermal losses, which in average for all the studied clusters is about $90 \%$. This high value, leads us to conclude that the viscous losses according to the homogenization theory is a valid and fast technique for the estimation of the total viscothermal losses expected from a cluster based on isotropic sonic crystals.

At this point it is interesting to study the origin of some features observed in the spectra of Fig. 9. For cluster
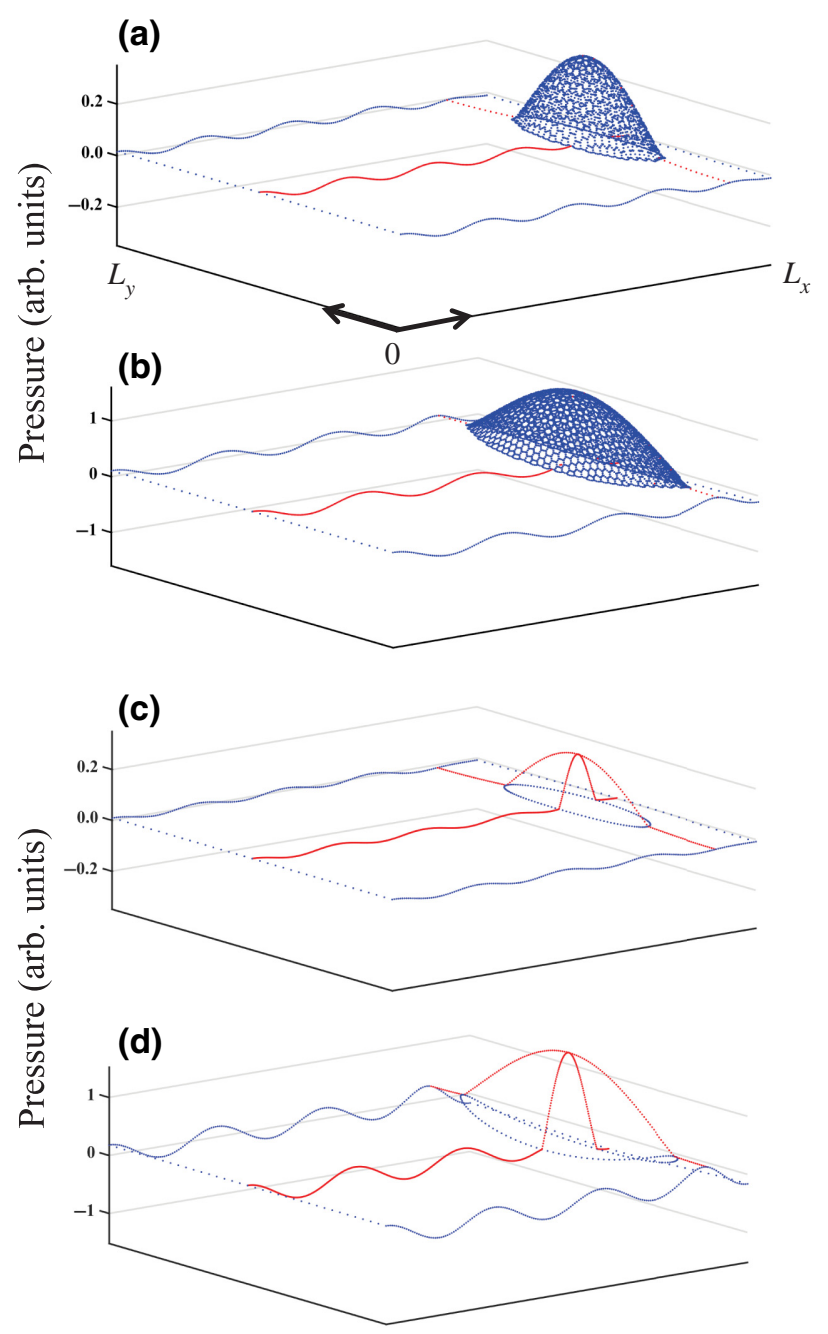

FIG. 10. (a),(b) Snapshots of the pressure patterns calculated at the frequencies of the fundamental modes of clusters A and B, respectively. (c),(d) Snapshots corresponding to the fundamental modes of the homogenized viscous cylinders representing clusters $\mathrm{A}$ and $\mathrm{B}$, respectively. The frequencies are defined by the positions of the arrows shown in Fig. 9. 
$\mathrm{A}$, which is the bare core of the $\mathrm{ABH}$, the absorbance peak located at $993.8 \mathrm{~Hz}$ corresponds to the excitation of its fundamental mode. A snapshot of the pressure amplitude corresponding to this mode is shown in Fig. 10(a). Remember that large pressure amplitudes associated with mode excitation are the origin of absorption enhancements in the absorbance spectra [22]. This effect is remarkable Fig. 9(b), corresponding to the absorbance spectra of cluster B. The strong peak appearing at $764.6 \mathrm{~Hz}$ corresponds to the excitation of its fundamental mode, which is represented in Fig. 10(b). This behavior is fairly well reproduced for the homogenized fluidlike cylinders, whose fundamental modes appear at 981.9 and $781.3 \mathrm{~Hz}$, respectively, for clusters A and B. Snapshots of the corresponding pressure patterns are depicted in Figs. 10(c) and 10(d), respectively.

The high accuracy of the homogenization approach allows the viscous absorbance to be studied as a function of the cluster size. Figure 11 shows the resulting dependence of the viscous quality factor with the number $N$ of cylinders in the cluster, which is considered as the more intuitive parameter describing the cluster size. For the smaller clusters, made of a few cylinders, the homogenization approach can be only applied to the "magic clusters" described in [43]. Seven magic clusters have been reported, all with hexagonal external shape and containing 7, 19, 37, 61, 85, 121, and 163 cylinders. The corresponding values are represented by symbols in Fig. 11 . For clusters containing more than about 200 cylinders, the homogenization approach works well in spite of the fact that sometimes their external shapes deviate slightly

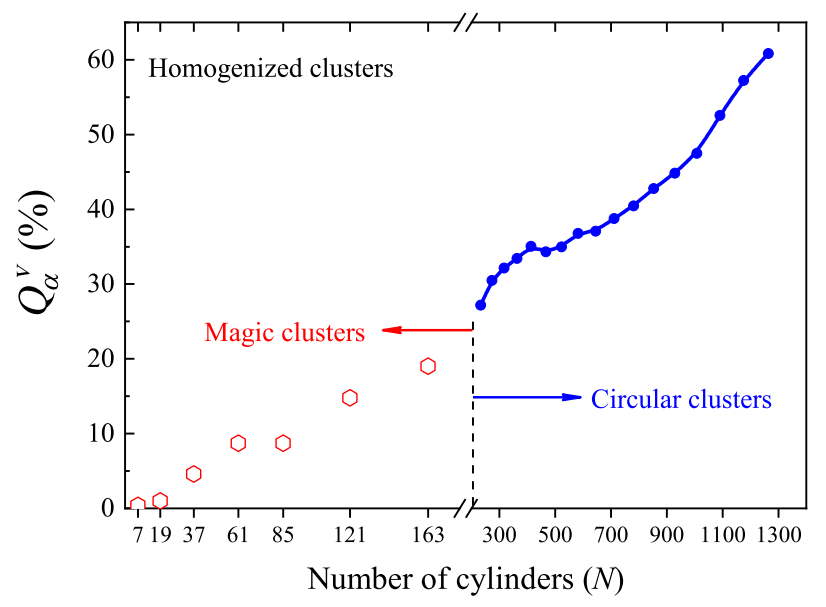

FIG. 11. The viscous quality factor $Q_{\alpha}^{v}$ (in \%) as a function of the number $N$ of cylinders in the cluster. For a given $N, Q_{\alpha}^{v}$ is determined from the absorbance spectra obtained for its correspondent homogenized viscous cylinders with circular shape. For the smaller clusters (hollow symbols), such correspondence is only allowed for certain magic clusters [43]. These results are valid for clusters in which the fraction of volume occupied by the cylinders is 0.836 . from the perfect circular shape. Figure 11 shows that viscous losses increase smoothly with increasing number of cylinders.

\section{SUMMARY}

We have comprehensively studied the absorbing properties of the 2D omnidirectional acoustic absorber presented in Ref. [7], here denominated as ABH. An improved BEM algorithm has been employed to calculate its absorbance spectrum due to viscothermal losses in a $2 \mathrm{D}$ chamber. The components of the $\mathrm{ABH}$ (i.e., the absorbing core and the GRIN lens shell) have been also separately analyzed in order to understand their contributions to the total absorption. The absorbance spectra predicted by the 2D BEM simulations show strong discrepancies with that reported experimentally in Ref. [7]. Regarding quality factors, the calculated values are always smaller than that obtained from the measurements. The observed disagreements are attributed to an artifact of the experimental setup, where the characterization chamber adds extra dissipation losses due to its imperfect manufacturing. This conclusion has been drawn from the analysis of the absorbance spectrum of the actual empty 3D chamber also presented here. In spite of the uncertainties associated with the measurements, the 2D BEM simulations reproduce fairly well the increase in the absorptive power of the bare core when it is surrounded by the GRIN lens in the ABH.

We have also demonstrated that the contribution of the viscous losses to the total viscothermal losses in the absorbing core of the $\mathrm{ABH}$ is about $90 \%$. The viscous dissipation has been studied with the homogenization procedure proposed in Ref. [29], which has been successfully validated here. It is shown that the effective parameters determined with the homogenization approach produce reliable results. The corresponding homogenized cylinders have been employed to calculate the viscous absorption of circular clusters as a function of the number of cylinders, and the results indicate that the viscous losses smoothly increase with the number of cylinders in the cluster. This homogenization procedure opens an easy way to tailor the viscous losses in clusters of hard cylinders.

\section{ACKNOWLEDGMENTS}

We thank Alfonso Climente for useful discussions. We also acknowledge Arkadii Krokhin and Jesús Arriaga for their help on the implementation of the homogenized viscosity.

\section{APPENDIX A: STANDING WAVES IN THE MULTIMODAL IMPEDANCE CHAMBER}

The actual MMIC employed in the experimental setup is a $3 \mathrm{D}$ cavity and the dimensions of its side lengths $L_{x}$, $L_{y}$, and $L_{z}$ are given in the caption of Fig. 2. Therefore, 
the standing waves excited in this cavity have discrete frequencies given by [39],

$$
f_{\ell m n}=(c / 2) \cdot\left[\left(\ell / L_{x}\right)^{2}+\left(m / L_{y}\right)^{2}+\left(n / L_{z}\right)^{2}\right]^{1 / 2},
$$

where $c$ is the sound speed inside the chamber and $(\ell, m, n)$ are integers taking values $0,1,2, \ldots, \infty$. Frequencies $f_{\ell m n}$ define the normal modes of this $3 \mathrm{D}$ cavity.

In practice, we are interested in frequencies below 3400 $\mathrm{Hz}$. In addition, the location of the speaker at the center of the left-hand side plate with rectangular section $L_{y} \times$ $L_{z}$ implies that only modes with even symmetry will be excited along the $y$ and $z$ axis, respectively.

By considering that the temperature inside the chamber is $20^{\circ} \mathrm{C}$, the corresponding sound speed $c=343.982 \mathrm{~m} / \mathrm{s}$ and, therefore, the cutoff frequencies for the onset of the first two modes $(0, m, 0)$ with an even number of nodal lines along the $y$ axis are

$$
\begin{aligned}
& f_{020}=\frac{c}{2} \cdot \frac{2}{L_{y}}=1162 \mathrm{~Hz}, \\
& f_{040}=\frac{c}{2} \cdot \frac{4}{L_{y}}=2324 \mathrm{~Hz},
\end{aligned}
$$

where modes with $m=2$ and 4 contain two and four nodal lines parallel to the $x$ axis, respectively. The mode $f_{060}$ has a frequency of $3486 \mathrm{~Hz}$, above the range of frequencies of interest here. Therefore, the linear expansion in Eq. (2) can be reduced to just three terms: those corresponding to $m=0,2$, and 4 .

The cutoff frequency for the excitation of the mode with two nodal lines $(n=2)$ in the vertical $z$ direction is

$$
f_{002}=\frac{c}{2} \cdot \frac{2}{L_{z}}=6948 \mathrm{~Hz}
$$

which is also above the frequencies of interest here and justifies the use of the simplified 2D cavity sketched in Fig. 2(b).

Figure 12(a) represents the frequencies of the standing waves excited in the $3 \mathrm{D}$ cavity employed as characterization chamber in the experimental setup. They are calculated using the analytical formula given in Eq. (A1): blue dots correspond to modes $(\ell, 0,0)$, green triangles correspond to modes $(\ell, 2,0)$ and red squares correspond to modes $(\ell, 4,0)$. Figure 12(b) plots the profiles of the modulus of coefficients $A_{m}$ calculated with the 2D BEM algorithm with viscothermal losses. They are obtained by solving the linear system of Eq. (2) in which the pressures $P_{\alpha}$ are recorded at the $\left(x_{\alpha}, y_{\alpha}\right)$ positions in the $2 \mathrm{D}$ cavity. Figure 12(c) shows the profiles corresponding to the coefficients calculated with the empty 3D cavity schematically represented in Fig. 2(a).

The profiles $\left|B_{m}\right|$ are not reported since they are practically indistinguishable from that of $\left|A_{m}\right|$. In other words,

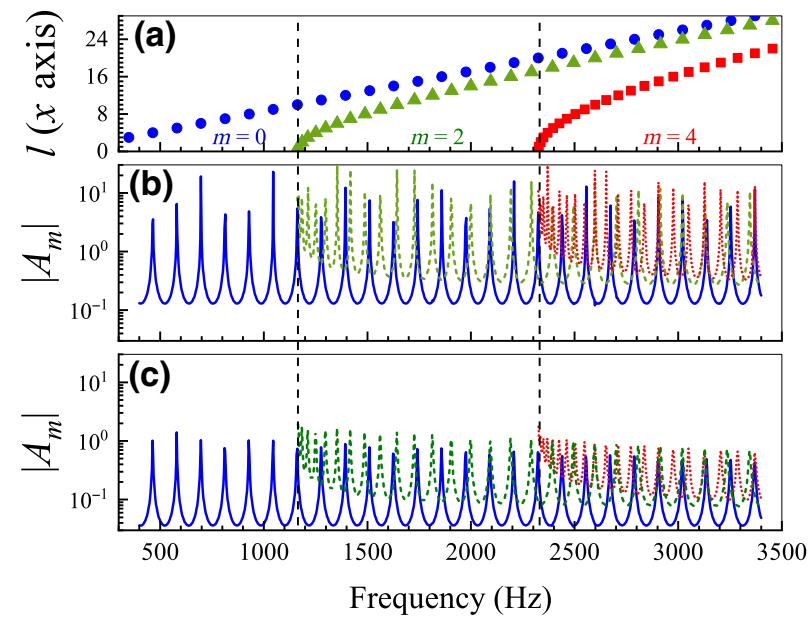

FIG. 12. (a) Calculated frequencies of the standing waves (modes) that can be excited in the $3 \mathrm{D}$ cavity defined by the empty MMIC employed in the experimental setup of Ref. [7]. (b) Modulus of coefficients $A_{m}$ calculated with the BEM algorithm using the empty 2D cavity depicted in Fig. 2(b). (c) The corresponding moduli calculated for the empty $3 \mathrm{D}$ cavity depicted in Fig. 2(a). Only the even modes $m=0$ (blue continuous lines), $m=2$ (green dashed lines), and $m=4$ (red dotted lines) are represented. The vertical dashed lines are guides for the eye and represent the onsets of modes $m=2$ and $m=4$.

when the cavity is empty, the BEM algorithm applied to both the $2 \mathrm{D}$ and the $3 \mathrm{D}$ cavities reproduces the expected result regarding the behavior of $A_{m}$ an $B_{m}$; they are practically the same according to the fact that the reflectance $\mathcal{R}$ is almost unity in spite of the dissipation losses on the walls. In addition, it is observed that the peaks of coefficients appear at the positions of the symbols depicted in Fig. 12(a). The accuracy of the BEM simulations is shown in Table II, where the first three rows provide the comparison between the frequencies of the standing waves inside the empty chambers calculated through Eq. (A1) (exact) and those extracted from BEM simulations applied to the 2D and $3 \mathrm{D}$ cavities. The lossless case reports the values calculated with no viscothermal losses in the BEM algorithm. The small discrepancies are mainly due to the fact that coefficients are calculated for discrete frequencies separated by intervals of $3 \mathrm{~Hz}$. It is concluded that viscothermal losses produce negligible effects on the standing waves excited in the 2D and $3 \mathrm{D}$ cavities and that, for the frequencies of interest, the actual 3D MMIC can be approached with a 2D cavity.

Table II also lists the frequencies of the peaks appearing on the profiles of coefficients $A_{m}$ and $B_{m}$ calculated with our BEM algorithm without losses when the $2 \mathrm{D}$ chamber contains the given structure. These frequencies represent the standing waves excited in the chamber with an object inside. For the frequencies (below $1 \mathrm{kHz}$ ) reported in the table, it is observed that the presence of a given structure produces small shifts in the frequencies of the standing 
TABLE II. Frequencies of standing waves (modes) excited in the MMIC employed in Ref. [7]. For the empty case, the exact values are obtained using the analytical expression given by Eq. (A1). The remaining frequencies correspond to the peaks' positions observed in the profiles of coefficients $A_{m}$ and $B_{m}$ calculated using the BEM algorithm with viscothermal losses. The second row reports values obtained from coefficients calculated using the empty 3D cavity depicted in Fig. 2(a). The third row corresponds to values obtained without viscothermal losses using the 2D cavity sketched in Fig. 2(b). The remaining rows are self-explained and are commented in the text.

\begin{tabular}{lcccccc}
\hline \hline Mode $(\ell m n)$ & $(400)$ & $(500)$ & $(600)$ & $(700)$ & $(800)$ & $(900)$ \\
\hline Empty (exact) & 465 & 581 & 697 & 813 & 930 & 1046 \\
Empty (3D) & 463 & 580 & 697 & 814 & 928 & 1045 \\
Empty (lossless) & 460 & 581 & 701 & 811 & 932 & 1042 \\
Empty & 466 & 580 & 697 & 814 & 929 & 1046 \\
ABH & 431 & 556 & 681 & 796 & 1004 & 1118 \\
EC & 431 & 556 & 681 & 806 & 900 & 1108 \\
Bare core & 442 & 556 & 681 & 806 & 921 & 1129 \\
GRIN shell & 442 & 567 & 671 & 733 & 837 & 1025 \\
\hline \hline
\end{tabular}

waves in the empty chamber. The size of the frequency shifts is affected by the introduction of an object in the cavity. This effect is more pronounced at high frequencies, as it is observed by comparing, for example, the profiles of the ABH coefficients shown in Fig. 4 with those shown in Fig. 12, corresponding to the empty 2D chamber.

\section{APPENDIX B: EXPERIMENTAL CHARACTERIZATION: CHAMBER ARTIFACTS ON THE MEASURED SPECTRA}

The experimental setup described in [7] involves a MMIC [see the sketch in Fig. 2(a)], which is constructed under the assumption that its absorbing behavior does not hinder the assessment of the structures in its interior. However, as we will see below, this expectation is not fulfilled.

A well-known artifact, appearing when the absorbing properties of a structure are measured inside a closed chamber, is the viscothermal losses taking place on the walls of the chamber. In other words, the measured absorbance of the structure is overestimated due to the fact that it is characterized inside a cavity. As the experimental setup employs a 3D cavity, one expects larger absorptions than that obtained with 2D BEM simulations just by the fact that the 3D chamber has additional walls. Fortunately, the calculation of the absorbance for the empty $3 \mathrm{D}$ cavity is feasible with the BEM algorithm and the results are discussed below.

Figure 13 shows the spectrum of the empty chamber obtained experimentally (symbols) and its comparison with that obtained using BEM simulations with viscothermal losses in the 3D cavity (blue solid line) and in the simplified 2D cavity (red dashed line). Both cavities are

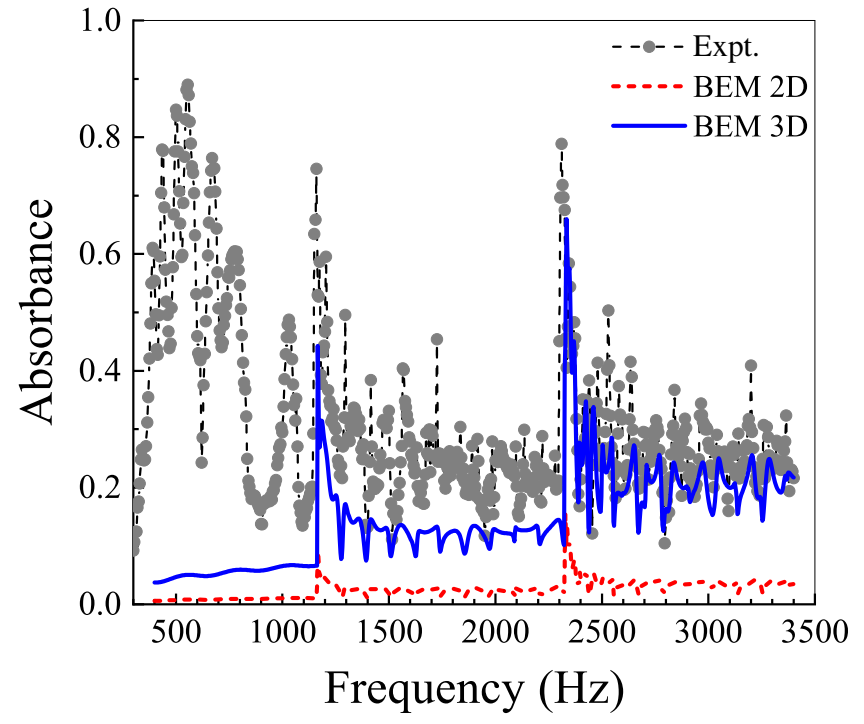

FIG. 13. Absorbance spectrum of the homemade MMIC measured with no object inside (symbols). The spectra calculated for the 2D empty chamber sketched in Fig. 3(b) (red dashed line) and that corresponding to the empty $3 \mathrm{D}$ box representing the actual MMIC (blue continuous line) are also shown.

sketched in Fig. 2. It is observed that the absorbance predicted by the 2D simulations is very small, always below 0.1 , though two strong peaks emerge at the frequencies where modes with two modal lines $(n=2)$ and four nodal lines $(n=4)$ start propagating. For the 3D simulations the absorbance increases significantly, achieving values up to 0.2 at the higher frequencies, where the three even modes coexist. As the viscothermal losses are due to particles impinging the chamber surfaces with oblique incidence, it can be concluded that the amount of particles with such property increases with the order of the modes propagating inside the chamber. Regarding the empty MMIC employed in the measurements, the measured absorbance spectrum (symbols) shows larger values than that obtained in the 2D and 3D BEM simulations, indicating the existence of measurement artifacts.

The strong absorbing features observed at low frequencies in the spectrum of the empty MMIC are attributed to imperfections in its construction, consisting of seven assembled aluminum plates $1 \mathrm{~cm}$ thick, one on them being removable to insert the samples in the chamber. In other words, for frequencies below $1160 \mathrm{~Hz}$, where the excited modes have perfect plane wavefronts, the pressure waves can excite flexural modes of the non-thick-enough plates employed in the construction of the actual 3D MMIC. This would explain the series of peaks repetitively observed not only in the empty chamber (see Fig. 13) but also in almost all the spectra measured in this study (see Figs. 5 and 6). For frequencies above $1160 \mathrm{~Hz}$, where modes with two and four nodal lines are allowed, the absorbing background due 
to the viscothermal losses on the walls is greatly enhanced due to the slits and pores existing in the imperfect joints between plates. Therefore, the absorptive effects occurring in the empty chamber appear as artifacts in the measured absorbance of the different structures.

Let us point out that the contribution of the chamber imperfections to the absorbing properties of the different structures is not well determined and could differ in the different measured spectra. The reason is that the measuring process involves several steps: (i) removing the plate defining the aperture to the chamber, (ii) inserting and centering the structure in the sample holder, and (iii) closing the aperture and sealing the chamber. At the end of the process, there is no guarantee that all the structures are measured under exactly the same conditions.

[1] E. Narimanov and A. Kildishev, Optical black hole: Broadband omnidirectional light absorber, Appl. Phys. Lett. 95, 041106 (2009).

[2] Q. Cheng, T. J. Cui, W. X. Jiang, and B. G. Cai, An omnidirectional electromagnetic absorber made of metamaterials, New J. Phys. 12, 063006 (2010).

[3] M. Yin, X. Y. Tian, L. L. Wu, and D. C. Li, A broadband and omnidirectional electromagnetic wave concentrator with gradient woodpile structure, Opt. Express 21, 19082 (2013).

[4] V. Krylov and F. Tilman, Acoustic "black holes" for flexural waves as effective vibration dampers, J. Sound Vib. 274, 605 (2004).

[5] R.-Q. Li, X.-F. Zhu, B. Liang, Y. Li, X.-Y. Zou, and J.C. Cheng, A broadband acoustic omnidirectional absorber comprising positive-index materials, Appl. Phys. Lett. 99, 193507 (2011).

[6] Q. Wei, Y. Cheng, and X. J. Liu, Acoustic omnidirectional superabsorber with arbitrary contour, Appl. Phys. Lett. 100, 094105 (2012).

[7] A. Climente, D. Torrent, and J. Sánchez-Dehesa, Omnidirectional broadband acoustic absorber based on metamaterials, Appl. Phys. Lett. 100, 144103 (2012).

[8] L.-Y. Zheng, Y. Wu, X.-L. Zhang, X. Ni, Z.-G. Chen, M.-H. Lu, and Y.-F. Chen, A new type of artificial structure to achieve broadband omnidirectional acoustic absorption, AIP Adv. 3, 102122 (2013).

[9] C. J. Naify, T. P. Martin, C. N. Layman, M. Nicholas, A. L. Thangawng, D. C. Calvo, and G. J. Orris, Underwater acoustic omnidirectional absorber, Appl. Phys. Lett. 104, 073505 (2014).

[10] A. S. Elliott, R. Venegas, J. P. Groby, and O. Umnova, Omnidirectional acoustic absorber with a porous core and a metamaterial matching layer, J. Appl. Phys. 115, 204902 (2014).

[11] Z.-M. Gu, Xue-Jiang, B. Liang, Y. Li, X.-Y. Zou, L.-L. Yin, and J.-C. Cheng, Experimental realization of broadband acoustic omnidirectional absorber by homogeneous anisotropic metamaterials, J. Appl. Phys. 117, 074502 (2015).
[12] A. Pelat, F. Gautier, S. C. Conlon, and F. Semperloti, The acoustic black hole: A review of theory and applications, J. Sound Vib. 476, 115316 (2020).

[13] A. Climente, D. Torrent, and J. Sánchez-Dehesa, Sound focusing by gradient index sonic lenses, Appl. Phys. Lett. 97, 104103 (2010).

[14] T. P. Martin, M. Nicholas, G. Orris, L. Cai, D. Torrent, and J. Sánchez-Dehesa, Sonic gradient index lens for aqueous applications, Appl. Phys. Lett. 97, 113503 (2010).

[15] J. Hyun, C.-S. Park, J. Chang, W.-H. Cho, and M. Kim, Gradient-index phononic crystals for omnidirectional acoustic wave focusing and energy harvesting, Appl. Phys. Lett. 116, 234101 (2020).

[16] C. Hu, B. Liang, J. Yang, and J. Cheng, Experimental demonstration of a three-dimensional omnidirectional and broadband acoustic concentrator using an anisotropic metamaterial, Sci. China-Phys. Mech. Astron. 64, 244304 (2021).

[17] M. A. Guild, V. M. García-Chocano, W. Kan, and J. Sánchez-Dehesa, Acoustic metamaterial absorbers based on multilayered sonic crystals, J. Appl. Phys. 117, 114902 (2015).

[18] T. W. Wu, Boundary Element Acoustics: Fundamentals and Computer Codes (WIT Press, Southampton, 2000).

[19] V. C. Henríquez, P. R. Andersen, J. S. Jensen, P. M. Juhl, and J. Sánchez-Dehesa, A numerical model of an acoustic metamaterial using the boundary element method including viscous and thermal losses, J. Comp. Acoust. 25, 1750006 (2017).

[20] V. C. Henríquez and P. M. Juhl, An axisymmetric boundary element formulation of sound wave propagation in fluids including viscous and thermal losses, J. Acoust. Soc. Am. 134, 3409 (2013).

[21] R. Graciá-Salgado, V. M. García-Chocano, D. Torrent, and J. Sánchez-Dehesa, Negative mass density and densitynear-zero quasi-two-dimensional metamaterial: Design and applications, Phys. Rev. B 88, 224305 (2013).

[22] V. C. Henríquez, V. M. Garcia-Chocano, and J. SánchezDehesa, Viscothermal Losses in Double-Negative Acoustic Metamaterials, Phys. Rev. Appl. 8, 014029 (2017).

[23] M. I. Hussein and M. J. Frazier, Band structure of phononic crystals with general damping, J. Appl. Phys. 108, 093506 (2010).

[24] I. E. Psarobas, Viscoelastic response of sonic band-gap materials, Phys. Rev. B 64, 012303 (2001).

[25] T. Frenzel, J. D. Brehm, T. Bückmann, R. Schittny, and M. Kadic, Three-dimensional labyrinthine acoustic metamaterials, Appl. Phys. Lett. 103, 061907 (2013).

[26] G. Theocaris, O. Richoux, V. Romero-García, and V. Tournat, Limits of slow sound propagation and transparency in lossy, locally resonant periodic structures, New J. Phys. 16, 093017 (2014).

[27] M. A. Guild, V. M. García-Chocano, W. Kan, and J. Sánchez-Dehesa, Enhanced inertia from lossy effective fluids using multi-scale sonic crystals, AIP Adv. 4, 124302 (2014).

[28] M. Molerón, M. Serra-García, and C. Daraio, Visco-thermal effects in acoustic metamaterials: From total transmission 
to total reflection and high absorption, New J. Phys. 18, 033003 (2016).

[29] M. A. Ibarias, Y. Zubov, J. Arriaga, and A. A. Krokhin, Speed of sound in periodic elastic composites, Phys. Rev. Res. 2, 022053 (2020).

[30] X. Zhang, Z. Qu, D. Tian, and Y. Fang, Acoustic characteristics of continuously graded phononic crystals, Appl. Acoust. 151, 22 (2019).

[31] A. Gueddida, Y. Pennec, S. Hémon, F. Lucklum, M. Vellekoop, N. Mukhin, R. Lucklum, B. Bonello, and B. D. Rouhani, in 2020 IEEE SENSORS (2020), p. 1.

[32] M. Kafesaki, R. S. Penciu, and E. N. Economou, Air Bubbles in Water: A Strongly Multiple Scattering Medium for Acoustic Waves, Phys. Rev. Lett. 84, 6050 (2000).

[33] A. A. Krokhin, J. Arriaga, and L. N. Gumen, Speed of Sound in Periodic Elastic Composites, Phys. Rev. Lett. 91, 264302 (2003).

[34] D. Torrent, A. Håkansson, F. Cervera, and J. SánchezDehesa, Homogenization of Two-Dimensional Clusters of Rigid Rods in Air, Phys. Rev. Lett. 96, 204302 (2006).

[35] D. Torrent and J. Sánchez-Dehesa, Effective parameters of clusters of cylinders embedded in a nonviscous fluid or gas, Phys. Rev. B 74, 224305 (2006).
[36] E. Reyes-Ayona, D. Torrent, and J. Sánchez-Dehesa, Homogenization theory for periodic distributions of elastic cylinders embedded in a viscous fluid, J. Acoust. Soc. Am. 132, 2896 (2012).

[37] A. D. Pierce, Acoustics: An Introduction to its Physical Principles and Applications (McGraw-Hill, New York, 1999).

[38] M. Bruneau, P. Herzog, J. Kergomard, and J. Polack, General formulation of the dispersion equation in bounded visco-thermal fluid, and application to some simple geometries, Wave Motion 11, 441 (1989).

[39] L. E. Kinsler, A. R. Frey, A. B. Coppens, and J. V. Sanders, Fundamentals of Acoustics (John Wiley \& Sons, New York, 1999).

[40] P. Morse and H. Feshbach, Methods of Theoretical Physics (Mc. Graw-Hill, New York, 1953).

[41] V. C. Henríquez and P. M. Juhl, in Proceedings of the Internoise 2010, Lisbon, Portugal (2010).

[42] D. Torrent and J. Sánchez-Dehesa, Acoustic metamaterials for new two-dimensional sonic devices, New J. Phys. 9, 323 (2007).

[43] D. Torrent, J. Sánchez-Dehesa, and F. Cervera, Evidence of two-dimensional magic clusters in the scattering of sound, Phys. Rev. B 75, 241404(R) (2007). 